\title{
GAD-Specific Cognitive Behavioral Treatment for Children and Adolescents: A Pilot Randomized Controlled Trial
}

\author{
Sean Perrin ${ }^{1,2,3}$ (D) Denise Bevan ${ }^{1,4} \cdot$ Susanna Payne ${ }^{1} \cdot$ Derek Bolton $^{1,2}$
}

Published online: 25 April 2019

(c) The Author(s) 2019

\begin{abstract}
Cognitive behavioral therapy (CBT) designed to target generalized anxiety disorder (GAD) in youth was examined in a pilot feasibility trial. Participants (aged 10-18 years) were randomized to either 10 weeks of individual CBT $(n=20)$ or supported wait-list $(\mathrm{n}=20)$. Diagnostic status (primary outcome) was assessed blindly at post-treatment for both groups, and at a 3-month follow-up for treated participants. Two participants failed to complete CBT and retained their GAD during the trial. Intention-to-treat analyses revealed large between-group differences in favor of CBT at post-treatment for remission from GAD ( $80 \%$ vs $0 \%)$ and comorbid disorders ( $83 \%$ vs $0 \%$ ), and for all secondary outcomes (child and parent-reported). All gains were maintained at 3-month follow-up in the CBT group. Consistent with the treatment model, significant pre- to post-treatment reductions in several cognitive processes were found for CBT but not wait-listed participants, with these gains maintained at follow-up. Further investigations are warranted. Trial registry: ISRCTN.com Identifier ISRCTN50951795
\end{abstract}

Keywords Generalized anxiety disorder · Cognitive behavior therapy · Laval model · Intolerance of uncertainty · Children · Adolescents

\section{Introduction}

Generalized Anxiety Disorder (GAD) is a chronic condition characterized by excessive and uncontrollable worry about a number of events and activities, accompanied by $\geq 3$ somatic symptoms (one for children) and clinically significant distress or impairment for no less than 6 months [American Psychological Association (American Psychiatric Association (APA), 2013]. The 12-month prevalence of GAD in children and adolescents aged 6-18 years (hereafter youth) in the community is $1-3 \%$, with affected individuals experiencing high rates of comorbidity, functional impairments, and a chronic course (Copeland et al. 2014). GAD is

Sean Perrin

sean.perrin@psy.lu.se

1 South London \& Maudsley NHS Foundation Trust, London, UK

2 Institute of Psychiatry Psychology \& Neuroscience, King's College London, London, UK

3 Present Address: Department of Psychology, Lund University, Box 213, 22100 Lund, Sweden

4 Present Address: Cumberledge Intermediate Care Centre, Southend-on-Sea, UK also one of the most commonly occurring comorbid disorders in youth seeking treatment for anxiety (Kendall et al. 2010). The evidence for the efficacy of transdiagnostic and GAD-specific treatments for GAD in youth is now briefly reviewed.

Cognitive behavioral therapy (CBT) and selective serotonin reuptake inhibitors (SSRIs) are the two most studied treatments for youth with GAD, either as a primary disorder or comorbid to another anxiety disorder (Wang et al. 2017). The most frequently evaluated CBT approaches, including the coping cat (CC) program (Kendall 1990), are transdiagnostic for anxiety disorders, not disorder-specific. The preponderance of transdiagnostic CBT programs for childhood anxiety reflects a general consensus that while specific anxiety disorders can be identified in youth, comorbidity is the rule rather than exception, and can be effectively treated with a broad-based protocol focused on 'anxiety' (cf., Hudson et al. 2015; Kendall et al. 2010; Waite and Creswell 2014).

Meta-analytic studies find that SSRIs and transdiagnostic CBT programs are superior to no treatment for youth with anxiety disorders, most frequently a combination of GAD, separation anxiety disorder, social anxiety disorder, and specific phobias (James et al. 2013; Wang et al. 2017). Approximately $60 \%$ achieve remission from at least one of their 
comorbid anxiety disorders, with gains maintained up to 36-weeks. As outcomes for GAD are not reported, the effectiveness of transdiagnostic approaches for this this condition remain unclear (Ewing et al. 2015). However, there is some evidence for the efficacy of the transdiagnostic approach Cool Kids (Lyneham et al. 2003). Hudson et al. (2015) combined data from controlled and uncontrolled trials (at total of 848 participants) of this approach and observed a $57.6 \%$ remission rate for GAD at 3-12 months post-treatment. This is consistent with response rates for GAD reported in several early cases studies involving treatments based on the CC approach (Eisen and Silverman 1993, 1999; Kane and Kendall 1989).

An empirical question arises whether outcomes for childhood GAD specifically can be improved by drawing upon models of GAD, most often developed with adults, to modify existing transdiagnostic CBT programs, or to develop new GAD-specific treatments. As an example of the former, Waters et al. (Waters et al. 2008) administered five sessions of CC (Kendall 1990) followed by five sessions of interpersonal therapy for adolescent depression (IPT-A; Mufson et al. 1994), to four adolescents with a primary diagnosis of GAD. All four participants were free of GAD at post-treatment, with clinically significant improvements on self-report measures of trait anxiety, depression, and interpersonal skills. The authors added IPT-A interventions based on the observation that excessive reassurance seeking from family members is common in those with GAD, and that interpersonal difficulties (generally) are more frequent in individuals with GAD compared to those with other anxiety disorders, and are associated with poorer outcomes from traditional CBT (Borkovec et al. 2002, 2003; Uhmann et al. 2010).

As an alternative approach to modifying existing transdiagnostic CBT programs for childhood anxiety, efforts have been made to develop and test GAD-specific CBT approaches (reviewed below) that draw upon a model of worry and GAD in adults (Laval model), developed by Dugas et al. (1998). Central to the model is the trait-like phenomenon intolerance of uncertainty (IU), originally described as "cognitive, emotional and behavioral reactions to uncertainty in everyday life situations" (Freeston et al. 1994, p. 792). The definition has evolved over time to include: "an individual's dispositional incapacity to endure the aversive response triggered by the perceived absence of salient, key, or sufficient, information, and sustained by the associated perception of uncertainty" (Carleton 2016, p. 31). When confronted with uncertainty, persons high in IU may engage strategies that make bouts of excessive worrying more likely or more difficult to stop (Dugas et al. 1998). These strategies, also mentioned in other models of GAD, include: a tendency to view uncertain outcomes as 'threats' that will either be difficult to prevent or to cope with if they occur (Negative Problem Orientation-NPO); the use of distraction and suppression to reduce intrusive images of the feared outcomes and distress (Cognitive Avoidance-CA); and dysfunctional appraisals about the nature of worries, including the belief that worrying may be necessary to prevent or lessen the impact of the feared outcomes (Positive Beliefs About Worry-PBW).

There is now a growing body of research on the applicability of the Laval model, and in particular-the central role played by IU, to the severity of childhood worry and anxiety. Laugesen et al. (2003) found that scores on measures of IU, CA, PBW, and NPO contributed significantly to the variance in total worry, with scores on the four measures correctly classifying $72.8 \%$ of adolescents (aged 14-18 years) into moderate and high worry groups. Using measures of IU, CA, and PBW, Fialko et al. (2012) fit a path model wherein IU acted as a higher-order vulnerability factor for CA and PBW, with the three variables together influencing the severity of self-reported worry and anxiety in adolescents (aged 13-19 years). The model fit in children (aged 7-12 years) had a similar hierarchical structure but without positive beliefs about worry. Donovan et al. (2016) found that IU, CA, NPO, PBW, and negative beliefs about worry accounted for $59 \%$ of the variance in worry in children (aged 8-12 year). However, only negative beliefs about worry and CA were unique predictors, accounting for $25 \%$ and $14 \%$ of the variance. In a longitudinal study of adolescents (mean age 12.5 years), Dugas et al. (2012) found that IU and worry interacted in a bidirectional and reciprocal relationship, with changes in IU producing changes in worry and vice versa. A recent meta-analysis of 31 studies examining the relationship between IU and worry, found that IU accounted for $39.7 \%$ of the variance in child worry and $35 \%$ of the variance in child anxiety (Osmanagaoglu et al. 2018). This (nonexhaustive) review, suggests that processes from the Laval model of worry may be important targets for intervention in the treatment of youth with GAD.

As originally developed for use with adults, the GADspecific treatment based on the Laval model involved modules targeting IU, PBW, NPO, and CA through worry-awareness training, exposure to situations involving uncertainty, modification of maladaptive beliefs about worries and the nature of everyday problems, and imaginal exposure to the content of worries (Dugas and Robichaud 2007). Trials of this treatment in adults report remission rates from GAD in the range of 70-88\%, with gains maintained up to 24-months (Ladouceur et al. 2000, 2004; Dugas et al. 2003, 2010). Consistent with the assumptions of the Laval model of worry, changes in IU during treatment for adults with GAD has been found to be associated with changes in worry; whether the treatment was based on the Laval model (Ladouceur et al. 2000) or not (Bomyea et al. 2015; Torbit and Laposa 2016). 
To date, three case series have been conducted that evaluate GAD-specific CBT approaches that target the four cognitive processes from the Laval model. Leger et al. (2003) administered an average of 13.2 sessions of CBT to four adolescents (aged 14-18 years; no drop-outs), 43\% of whom were free of their GAD diagnosis at post-treatment, declining to $28.6 \%$ at the 12-month follow-up. Payne et al. (2011) modified this treatment for use with children and younger adolescents. After an average of 9.7 sessions (and no dropouts), $81 \%$ of the 16 participants (aged 7-17 years) were free of their GAD diagnosis, with 59\% losing their comorbid anxiety and depressive disorders, and large effect size reductions in child-reported worry and anxiety. Wahlund et al. (2019) administered a 12-session, IU-focused CBT program to 12 adolescents (aged 13-18 years; no drop-outs) with excessive worry, nine of whom had GAD. The adolescentfocused sessions were supplemented by an internet-delivered program for parents designed to teach them about worry, IU, and helpful parenting behaviors. At post-treatment, $58.3 \%$ of the adolescents were rated as much or very much improved by independent clinicians, rising to $66 \%$ at the three-month follow-up. Moderate to large effect sizes were observed for adolescent-and parent-reported worry, anxiety, depression and global functioning at post-treatment (maintained at follow-up). Moderate to large effect-size reductions were also reported for self-report measures of IU, NPO, CA, and PBW, although the pre-to-post-treatment changes for PBW were non-significant.

To date, there has been one RCT of a GAD-specific treatment for youth. Holmes et al. (2014) evaluated the efficacy of a group-based, child- and parent-focused, CBT program targeting the four cognitive processes from the Laval model, as well as perfectionism and sleep difficulties. Participants were 42 children aged 7-12 years with a primary diagnosis of GAD, randomized to 10 -weeks of the group treatment plus two 90-min booster sessions, or a 12-week wait-list. Parents completed a parallel, 7-session group. Three of the 20 participants (15\%) in the CBT condition dropped out. Based on intention-to-treat analyses, $52.9 \%$ of the children in the CBT group were free of their GAD (and 17.6\% free of all diagnoses) at post-treatment, rising to $80 \%$ at threemonth follow-up. None of the children in the wait-list group lost their GAD or comorbid diagnoses. At post-treatment, there were no significant within-subject or between-group changes on child-report measures of IU, CA, NPO, and PBW. However, at follow-up, and relative to baseline, significant reductions in the moderate to large range were observed for treated participant's scores on the measures of IU, CA, NPO-but not PBW.

The current study was designed as a randomized controlled, pilot feasibility trial with the primary aim of assessing the tolerability and efficacy of a child-friendly adaptation of the GAD-specific CBT approach described in Dugas and Robichaud (2007). This would be only the second randomized control trial of a GAD-specific treatment with children and the first to include adolescents. As such, the design incorporated a delayed-treatment (wait-list) condition as the control group. Based on the results of a previous uncontrolled trial of the current treatment approach (Payne et al. 2011), as well as outcomes reported in the literature for GAD-specific treatments for youth (described above), we anticipated that the current treatment would be well tolerated (i.e., $\leq 10 \%$ drop-outs), and would yield differences in GAD remission relative to wait-list controls in excess of $50 \%$ at post-treatment. Based on the results from the two GAD-specific treatment trials that administered measures of IU, CA, NPO, and PBW (Holmes et al. 2014; Wahlund et al. 2019), we anticipated moderate to large effect-size differences for the four cognitive measures between CBT and wait-list at post-treatment, and between pre-treatment and post-treatment/follow-up for treated participants.

\section{Method}

\section{Participants}

The 40 participants (aged 10-18 years) were recruited from standard referrals to the 10 child and adolescent mental health services and a specialist child anxiety disorders clinic affiliated with the South London and Maudsley NHS Foundation Trust (United Kingdom). The Trust serves a catchment area of approximately 1.3 million inhabitants from diverse socioeconomic and ethnic backgrounds. Referrals may be initiated by the parent (or adolescent), family doctor, school, or social services; all clinical care is free of charge.

Inclusion criteria for the trial were as follows: (1) Aged 10-18 years; (2) referred for treatment of anxiety; (3) a current, primary diagnosis of DSM-IV GAD; (4) no other psychiatric problems in need of more urgent treatment (including self-injurious thoughts/behaviors or substance use/ abuse); (5) no concurrent psychological or pharmacological treatment for any disorder; and (6) the absence of moderate to severe learning difficulties as evidenced in the medical or school records or as reported by the referrer/parent at the pre-trial screening. No other inclusion/exclusion criteria were applied. All participants were fluent in English.

\section{Procedure}

The study was planned as a Phase 0/1 RCT with the participants randomly allocated, in equal proportions (no stratification), to either 10 weeks of either individual, GAD-specific cognitive behavior therapy (CBT) or a supported wait-list (WL). Participants in both groups completed primary and secondary outcome measures at pre- and post-treatment/ 
wait-list. Wait-listed participants who still required treatment at the end of the 10-week wait-list were provided immediate treatment in the clinic, either the GAD-specific CBT tested in this trial or another appropriate treatment (these data are not included in this study). CBT participants (only) were reassessed at a 3-month follow-up.

Following referral, an initial phone screening was carried out to determine if the primary complaint was GAD, and if positive, written information about the trial was sent to the family, along with a date for a face-to-face assessment with the trial coordinator. All participants attended the face-to-face assessment with at least one parent. If all inclusion criteria were met at the face-to-face assessment, the trial coordinator contacted the lead author who accessed an online, random allocation programme created by King's College London Clinical Trials Unit for the purposes of this trial. The trial coordinator revealed the allocation to the family and told them they could withdraw from the trial at any time without negatively impacting their access to treatment. At all stages, clinical need overrode the trial protocol.

Figure 1 presents the CONSORT diagram of participant flow through the trial. Complete data were available for 38 of the 40 study participants (95\%). All WL participants $(n=20)$ completed the 10 -week wait-list, including all diagnostic interviews, secondary outcome measures, and cognitive process measures at pre- and post-wait-list. All but two of the CBT participants (both adolescents) completed treatment. One CBT participant dropped out after four sessions because they wanted to focus on social anxiety and not $\mathrm{GAD}$, and another was removed from the trial after four sessions because of the onset of suicidal thoughts in response to a family crisis that began after the trial treatment had commenced. This crisis was unrelated to the participant's GAD or treatment. While both non-completers were out of the trial, they continued to receive treatment in the clinic where the trial was carried out. The investigators were aware that they continued to suffer from GAD at the time that their (perprotocol) post-treatment and 3-month follow-up assessments would have occurred. Thus, their pre-treatment data (all) was carried forward to post-treatment and follow-up. Data for all primary, secondary, and cognitive process measures administered at pre-, post-treatment, and 3-month-follow-up were available for the remaining $18 \mathrm{CBT}$ participants.

\section{Measures}

\section{Primary Outcome}

The primary outcome was defined as the difference in the proportion of participants with a GAD diagnosis in the CBT versus WL groups at post-treatment/wait-list. GAD was assessed with the Anxiety Disorders Interview Schedule for DSM-IV, Child and Parent Report Version (ADIS-C/P;
Silverman and Nelles 1998). All participants, and at least one parent/primary caregiver, were interviewed separately with ADIS-C/P and the information combined to arrive at the diagnostic profile and the clinician's separate severity ratings $(0-8)$ for GAD and any comorbid disorders. ADIS$\mathrm{C} / \mathrm{P}$ interviews were carried out by fully qualified, doctorallevel clinical psychologists with extensive training in the use of the ADIS-C/P in clinical and research contexts. Assessors of the primary outcome at post-treatment, and for CBT participants only-the 3-month follow-up, were independent of the trial and the clinic where the trial was carried out. The assessors were blinded to the participant's treatment allocation and history. Participants (and their parents) were given written instructions just prior to the post-treatment/wait-list and follow-up assessments not to disclose their treatment history.

\section{Secondary Outcomes}

In order to assess a broader range of outcomes than remission from GAD, several child-, parent-, and cliniciancompleted measures of symptoms, overall functioning, and quality of life, were administered to all participants at pre- and post-treatment/wait-list, and for CBT participants (only) at the 3-month-follow-up. The secondary outcome measures have been validated for use with children and adolescents, have excellent psychometric properties, and are routinely used in trials of anxiety disordered youth. The secondary outcome measures were as follows: (1) Worry Severity (child-report): Penn State Worry Questionnaire for Children (PSWQ-C; Chorpita et al. 1997); (2) Anxiety/ GAD Symptoms (child and parent-report): Screen for Child Anxiety Related Emotional Disorders-Child and Parent Report Version (SCARED-C/P; Birmaher et al. 1997); (3) Depression (child and parent-report): Mood and Feelings Questionnaire-Child and Parent Report Versions (MFQ-C/P; Angold et al. 1995); (4) Conduct Problems, Peer Difficulties, Overall Impairment (parent-report): Strengths and Difficulties Questionnaire-Parent Version (SDQ-P; Goodman 1997); (5) Global Functioning (clinician-report): Children's Global Assessment Scale (CGAS; Shaffer et al. 1983); and (6) Quality of Life (child- report): Pediatric Quality of Life Enjoyment and Satisfaction Questionnaire (PQ- LES-Q; Endicott et al. 2006).

\section{Cognitive Processes from the Laval Model of Worry}

At the time this trial was planned, measures of the four cognitive processes from the Laval model of worry (IU, CA, NPO, PBW), adapted and formally validated for use with children and adolescents, did not exist. For the purposes of this trial, a five-item version of each of the four self-report measures used by Dugas et al. (1998) to assess IU, CA, NPO, 
Fig. 1 CONSORT flow chart

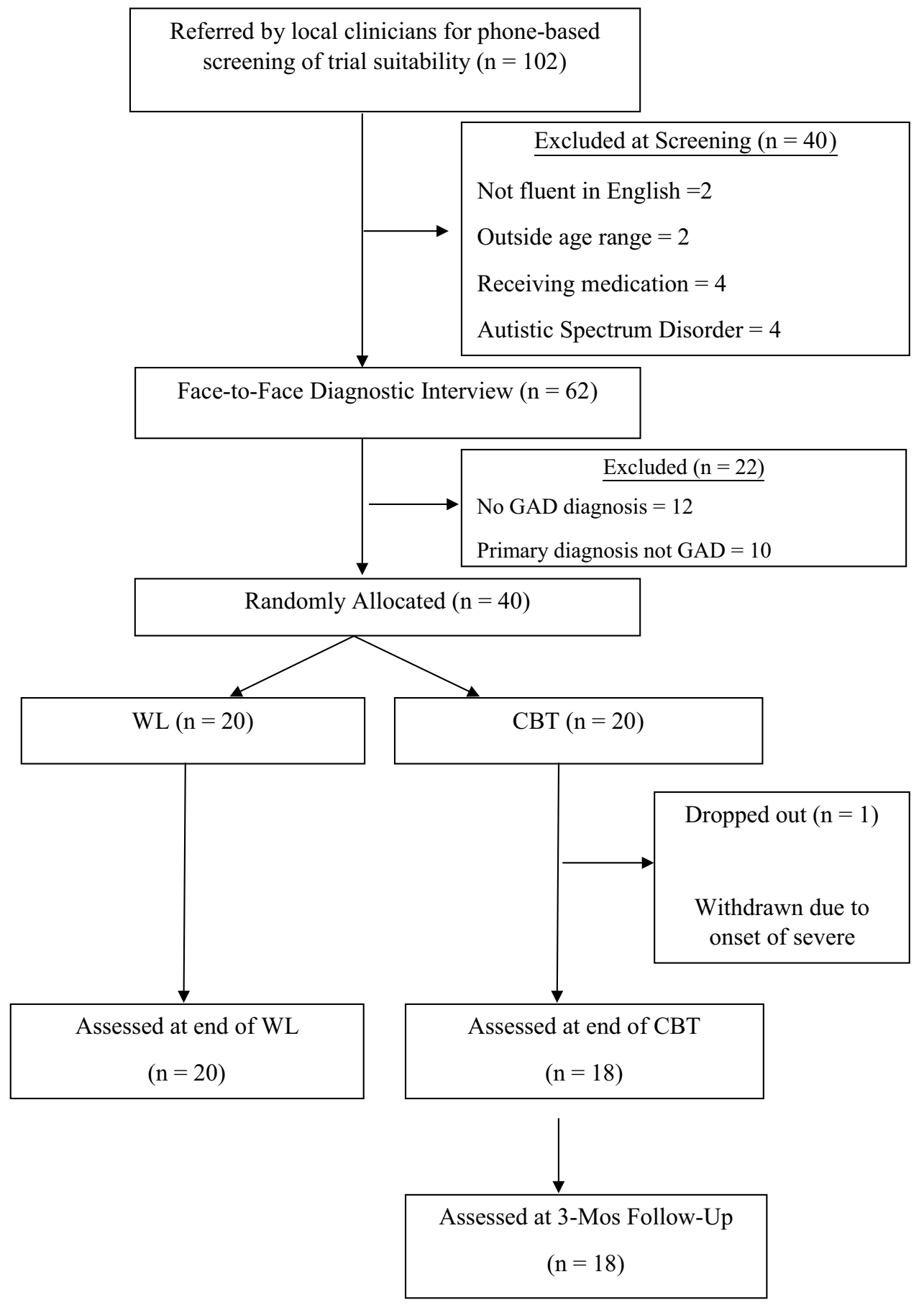

and PBW in adults were created (Fialko et al. 2009; Fialko et al. 2012). Briefly, for each of the four scales, five items were chosen from the original questionnaire based on these items having the highest item-total correlations, or itemsubscale/factor loadings for measures with subscales. The five-item scales were then given to clinically-referred and non-referred children and adolescents, and small changes made to increase comprehensibility. The five-item measures of IU, CA, and PBW were also administered to 515 British youth (aged 7-19 years) and found to have high levels of internal consistency, similar factor structures as the fulllength originals, and to correlate (positively) in the moderate to large range with self-reported worry and anxiety (Fialko et al. 2012). Swedish-language versions of the same fiveitem cognitive process measures used in this trial were found to be sensitive to the effects of an IU-focused CBT program for excessive worry in adolescents (aged 13-18 years) (Wahlund et al. 2019). 
Intolerance of Uncertainty (IU) was assessed using the child-report, Brief Intolerance of Uncertainty Scale (BriefIUS; Fialko et al. 2012). The scale is comprised of the following five-items from the 27-item IUS for adults (Freeston et al. 1994): Not knowing what may happen next makes my life horrible; I can't be relaxed if I don't know what will happen tomorrow; When I am not sure about something, I can't get on with it; When I am not sure what will happen next, I can't do things very well; Not knowing what may happen next can make me scared or sad. Each item is rated on a 5 -point scale $(1=$ Not all like me; $5=$ Completely like me); higher total scores indicate higher IU. The internal reliability coefficients for the Brief-IUS for the 40 participants in this study were: pre-treatment/wait-list $\alpha=.89$; post-treatment/ wait-list $\alpha=.95$.

Cognitive Avoidance (CA) was assessed using the Brief Cognitive Avoidance Questionnaire (Brief-CAQ; Fialko et al. 2012), comprised of the following 5-items from the 25-item CAQ for adults (Sexton and Dugas 2008): There are things that I would rather not think about; I have thoughts that I try to avoid; To avoid thinking about things that upset me, I force myself to think about something else; I avoid doing anything that reminds me of things I don't want to think about; When I have a scary picture in my mind, I say things to myself in my head to replace the picture. Each item is rated on a 5 -point scale ( $1=$ Never; $5=$ always); higher total scores indicate higher CA. Internal reliability coefficients for the Brief-CAQ for the 40 participants in this study were: pre-treatment/wait-list $\alpha=.83$; post-treatment/wait-list $\alpha=.90$.

Positive Beliefs About Worry (PBW) were assessed using the Brief Why Worry Scale-II (Brief-WW-II; Fialko et al. 2012) comprised of the following 5 -items from the 25 -item WW-II for adults (Holowka et al. 2000): If I worry in advance, I will be less upset if something bad happens; Worrying can stop bad things from happening; Worrying helps me find a better way to do things; Worry helps me to get started on things I must do; The fact that I worry shows that I am a good person. Each item is rated on a 5-point scale ( $1=$ Not all true of me; $5=$ Completely true of me); higher total scores indicate higher PBW. Internal reliability coefficients for the Brief-WW-II for the 40 participants in this study were: pretreatment/wait-list $\alpha=.77$; post-treatment/ wait-list $\alpha=.79$.

Negative Problem Orientation (NPO) was measured with the Brief Negative Problem Orientation Questionnaire (Brief-NPOQ, unpublished measure), comprised of fiveitems drawn from the 12-item NPOQ for adults (Robichaud and Dugas, 2005): I often doubt my ability to solve problems; Often my problems seem unmanageable; When I try to solve a problem I often question my own ability; I often get the impression that my problems can't be solved; My first reaction to a problem is to question my own ability.
Each item is rated on a 5-point scale $(1=$ Not all true of me; $5=$ Completely true of me); higher scores indicate higher NPO. This scale has not been formally validated. However, the five items overlap with those from five-item measure of NPO used in the trial by Holmes et al. (2014), which found the NPO measure to be sensitive to the effects of a GADspecific treatment based on the Laval model in children (aged 7-12 years). Internal reliability coefficients for the scale in the 40 participants in the present study were: pretreatment/wait-list $\alpha=.93$; post-treatment/wait-list $\alpha=.96$.

\section{Treatment Protocol}

\section{Cognitive Behavioral Therapy for GAD}

The treatment was a child-friendly adaptation of the CBT for GAD approach described in Dugas and Robichaud (2007). As described by Dugas and Robichaud (2007), treatment proceeds sequentially through six stages/modules: (1) worry awareness training; (2) planned exposure to uncertainty; (3) modification of dysfunctional beliefs about worry; (4) modified problem-solving training; (5) imaginal exposure to unpleasant images or worries; and 6) relapse prevention. The treatment was successfully piloted with seven adolescents (aged 14-18 years) with GAD by Leger et al. (2003).

The modular approach and same interventions as described in Dugas and Robichaud (2007) were retained in the current trial, but the treatment was modified with the aims of making it less abstract and more engaging for children and younger adolescents who might not immediately grasp the relevant processes/concepts (IU, CA, NPO, PBW). To achieve these aims, the therapist would always use language commensurate with the young person's age and vocabulary. Second, at the start of each module, therapistled explanations about the cognitive processes and module were kept very brief, always confined to the beginning of a single session. It was explained to the young person that they would learn the relevant concepts more easily through the discussion of a recent worry, behavioral experiments, and imaginal exercises.

Third, the therapist would then elicit a concrete, specific and personalized example of the young person's worrying from the past week, and use this example to help them notice "real-time" instances of the cognitive process to be targeted in that module, or if necessary because the child could not identify the cognitive process relevant to that module, a cognitive process from another module. For example, if during an exploration of concrete episodes of worry during the third module, the child was unable to identify any dysfunctional beliefs about their worries, the therapist could shift the focus to appraisals about uncertainty, negative problem orientation, cognitive avoidance, or their tolerance for uncertainty and distress related to feared outcomes. 
In practice, most participants (regardless of age) were able to progress through the modules as described in Dugas and Robichaud (2007). However, some participants (both children and adolescents) were not able to identify dysfunctional beliefs about worry, and rather than repeatedly engaging the participant in Socratic questioning or other exercises to elicit such beliefs, the therapist had the flexibility to move to the next or previous modules as needed.

Just prior to session 1, the child completed the self-report measure of worry (PSWQ-C), followed by a discussion about the nature of worry/GAD and its treatment, the role played by the four cognitive processes play in the maintenance of worry/GAD, and the importance of self-guided exposure to situations involving uncertainty. Thereafter, each session began with the child completing the worry measure and a brief review of their progress over the past week. From session two onwards, the therapist proceeded through the modules in the sequence described above, but in every session the therapist would elicit a concrete episode of worry from the past week that was then tied to behavioral experiments and imaginal exposures, as described in Dugas and Robichaud (2007), and with the purpose of: (a) increasing awareness about worry; (b) increasing tolerance for uncertainty; (c) reducing cognitive avoidance; (c) modifying positive beliefs about worry; (d) helping the young person distinguish between genuine problems from worries and to modify negative beliefs about their ability to solve their problems; and (e) testing the young person's tolerance for uncertainty and feelings of fear in the event of a possible but unlikely feared outcome.

Homework tasks largely followed those described in Dugas and Robichaud (2007): (1) increasing worry awareness by pausing several times each day to reflect upon, write down, and distinguish between worries about current problems versus hypothetical situations; (2) to plan confrontations each day with situations that involve uncertainty and normally trigger worries (e.g. raising your hand in class when you are not $100 \%$ sure of the answer); (3) reducing requests for reassurance from parents/others; (4) practicing behavioral experiments to test dysfunctional beliefs about IU, CA, NPO, and PBW; and (5) engaging in self-guided exposures to the content of worries to test their ability to tolerate uncertainty and the distress. Homework assignments were always discussed in session and reflected experiments/ exposures practiced in the session.

Parents were invited to join session one and asked to provide praise/rewards to their child for attempting to complete homework assignments. They were not asked to guide the child in homework exercises or to challenge their child's maladaptive beliefs about uncertainty, the likelihood of feared outcomes, the child's problem-solving abilities, or the worries themselves. The concept of secondary gain was introduced such that requests from the child for reassurance about worries can sometimes result in parents trying to alleviate their child's distress through the provision of rewards or allowing the child to escape from activities that may have prompted a bout of excessive worrying. The child and parents were encouraged to be aware of these contingencies, and the parents to refrain from rewarding avoidance and reassurance seeking. No further guidance was provided to the parents about dealing with their child's worries or reassurance-seeking.

Sessions 2-10 were one-to-one with the participant, not all of whom were brought to the clinic by their parents for every session. Where there was time and the child agreed, the parents were invited to join for the last 5-10 min of the session to discuss progress. If the therapist or parents wanted a separate meeting one could be scheduled, however, no such meetings were requested by the parents or deemed necessary by the trial therapists. Prior to the start of session 5 (midtreatment), the child and parents completed several of the baseline symptom questionnaires and progress in treatment discussed with the child and parent separately and together during the session.

\section{Therapist Training and Treatment Adherence}

All treatment was provided by two of the authors (Payne and Bevan) under the supervision of the lead author. Both trial therapists were fully qualified doctoral-level clinical psychologists at the time of the trial. Their core professional training focused on cognitive and behavioral interventions. Both had 2-3 years of post-doctoral experience providing CBT interventions to children and/or adults with GAD and other anxiety disorders, under supervision from internationally recognized experts in CBT for child/adult anxiety.

Prior to the trial, Payne and the lead author attended a 1-day clinical workshop by Dugas on the CBT for GAD approach described in Dugas and Robichaud (2007). After the treatment manual was adapted by the authors for use with children and adolescents (described above), it was successfully piloted with 16, clinically-referred youth (aged 7-18 years) with a primary diagnosis of GAD (Payne et al. 2011), with Payne as the sole therapist under the supervision of the lead author. Prior to treating cases in the current trial, Bevan was provided with training in the treatment model/manual by Payne and the lead author, and then observed Payne treat five young people with GAD using the manual. Bevan then treated five youth with GAD who were not included in the current trial using the treatment manual under observation/supervisions from Payne and the lead author.

Treatment adherence was monitored through supervision with the lead author with reference to the trial manual. When permission was given by the participant/ parent, a video was made of the therapy session for the 
purposes of supervision. The videos were used in supervision to facilitate therapist adherence to the manual. Videorecordings were not available for all sessions and were not formally rated for adherence.

\section{Treatment Credibility and Engagement}

At the end of session 2, CBT participants were given a 4-item measure of treatment credibility and therapeutic engagement to complete at home and return to the trial coordinator by post. The participants were told the therapist would not see the completed measure or be informed of the child's views. The measure was developed for the purpose of this trial and based on the widely-used, 6-item Credibility Expectancy Questionnaire developed by Devilly and Borkovec (2000) used in clinical trials with adults. Prior to the trial, the 4-item measure was administered to 10 children and adolescents (aged 10-14) receiving CBT for an anxiety disorder and feedback obtained on the comprehensibility of the items and the rating scale. As a result of the feedback we added the phrase ('makes sense') to the first item (see below). The first three questions address treatment credibility: "Does this treatment seem logical (make sense) to you?" ( $1=$ Not at all, 10= Makes complete sense); "How certain are you that this treatment will be helpful for your problems?" $(1=$ Not at all certain, $10=$ Completely certain); "How confident would you feel recommending this treatment to a friend with the same type of problems as you?" $(1=$ Not at all confident, $10=$ Completely confident $)$.

The last question addresses therapeutic engagement: "Some people think that the following things are important in therapy sessions: (1) Your therapist is warm and supportive; (2) Your therapist is helping you; (3) You and your therapist get on well; (4) You feel your therapist listens to what you have to say and treats what you say as important; (5) You and your therapist are working together as a team; (6) You and your therapist share the same way of thinking about the problem; (7) You feel that what your therapist is suggesting will be helpful. Overall, how much do you think this has been happening in your sessions so far? Please circle the number that you think is closest to how you feel" ( $1=$ Not at all, $10=$ Very much)."

At the end of session 2, CBT participants rated the therapy as: logical/sensible $(\mathrm{M}=8.0, \mathrm{SD}=1.5, \mathrm{Min} /$ $\mathrm{Max}=5 / 10)$; likely to succeed $(\mathrm{M}=8.3, \mathrm{SD}=1.6$, Range $=6 / 10)$; recommendable to a friend with the same problem $(M=8.5, S D=1.5$, Range $=4 / 10)$; and the therapist as warm/engaged $(M=8.9, S D=1.0$, Range $=7 / 10)$. The internal reliability coefficient for total scores on the 4-item scale in the treated participants $(\mathrm{N}=20)$ was $\alpha=.78$.

\section{Wait-List Condition}

At the time of allocation, wait-listed participants were provided information about the prevalence of worry and GAD, 10 copies of the self-report measure of worry (PSWQ-C), along with pre-paid envelopes addressed to the trial coordinator, and the date of their first treatment appointment after the 10 -week wait-list ended. No information about causes or maintaining factors in relation to worry/GAD, or anxiety broadly, were discussed or presented in written form. The wait-listed participants were asked to complete the worry measure at the end of each week and to return it by post to the trial coordinator. No other measures were completed by the wait-listed participants during weeks $2-9$ of the wait-list. The participant and their parents were told that they would receive one phone call per week from the trial coordinator to ask them how they were coping, to remind them of the date of the upcoming treatment appointment, and to complete/ return the weekly worry measure. No advice about managing worries or any other difficulties were provided during these phone calls.

\section{Statistical Analyses}

Sample size was calculated a priori based on the difference between the proportion of participants in the CBT and WL group with GAD being $\geq 50 \%$, with $\leq 10 \%$ drop-outs. To achieve a proportional difference this size, with $80 \%$ power, and $p=0.05$, required a minimum of 14 participants per group. To account for possible drop-outs, recruitment was set at 20 participants per group. All analyses and reporting were planned on the 'intention-to-treat' (ITT) principle. Data for the primary outcome variable was missing for only two trial participants both in the CBT group. However, as both continued to receive treatment from the clinic where the trial was carried out, their status with respect to the primary outcome was known to the authors. With this information, and in consultation with a clinical trials unit, we used a lastobservation-carried-forward strategy (LOCF).

For categorical outcomes, $2 \times 2$ chi squares were used to assess the proportion of CBT and WL participants remitted from their GAD diagnosis (primary outcome) and any comorbid disorders (secondary outcome) at post-treatment. For the remaining secondary outcomes (continuous), we were primarily interested in group differences at posttreatment and carried out one-way analyses of covariance (ANCOVA) with group (CBT vs WL) as the between factor, post-treatment scores as the dependent variable, and pretreatment scores on the same measure as the covariate.

Within-subject analyses were carried out for participants in the CBT group as they underwent a further assessment at 3-month follow-up. The presence versus absence of GAD and any comorbid disorders at post-treatment versus 
3-month-follow-up were examined using $2 \times 2$ chi squares. For all continuously-measured secondary outcomes and the cognitive process measures, one-way, repeated measure analyses of variance (ANOVA) with three timepoints (pre-, posttreatment, 3-month follow-up), and simple contrasts, were carried out. Between-group and within-subject effect sizes for all (continuous) outcomes and cognitive process measures are reported as partial eta-squared $\left(\eta^{2}=\right.$ sum of squares for the measure/sum of squares for the measure + sum of squares for error). Partial eta squared $\left(\mathrm{n}^{2}\right)=.02, .13$ and .26 reflect small, medium, and large effect sizes (Cohen 1988).

\section{Results}

\section{Baseline Characteristics of CBT and WL Participants}

Table 1 presents the baseline characteristics separately for participants in the CBT and WL groups. Between-group comparisons for the CBT and WL on the baseline characteristics in Table 1 were evaluated using $2 \times 2$ Chi squares and t-tests. No significant between-group differences were observed for any of the listed variables (or for the mean number of disorders), except that more CBT participants had comorbid Separation Anxiety Disorder $\left(\chi^{2}(1)=4.8\right.$, $\mathrm{p}=.028)$.

Not reported in Table 1, there were no between-group differences in respect of the proportion of participants aged $10-12$ years $(\mathrm{CBT}=55 \%$; $\mathrm{WL}=35 \%)$ or the mean number of current DSM-IV diagnoses (including GAD) $(\mathrm{CBT}=1.7$ $(\mathrm{SD}=.73) ; \mathrm{WL}=1.6(\mathrm{SD}=.59)$. Youth with moderate to

Table 1 Demographic and clinical characteristics of participants by trial arm at baseline

\begin{tabular}{lll}
\hline Participant characteristics & $\begin{array}{l}\text { CBT } \\
(\mathrm{n}=20)\end{array}$ & $\begin{array}{l}\text { Wait-list } \\
(\mathrm{n}=20)\end{array}$ \\
\hline Age, mean (SD) & $13.2(2.4)$ & $13.6(2.8)$ \\
Female, \% (n) & $55(11)$ & $70(14)$ \\
Ethnic minority, \% (n) & $25(5)$ & $30(6)$ \\
Comorbid disorder(s) present, \% (n) & $60(12)$ & $55(11)$ \\
Separation anxiety disorder & $40(8)$ & $10(2)$ \\
Social phobia & $10(2)$ & $35(7)$ \\
Specific phobia & $5(1)$ & $0(0)$ \\
Major depression & $15(3)$ & $15(3)$ \\
Previous mental health treatment, \% (n) & $45(9)$ & $40(8)$ \\
For GAD & $10(2)$ & $10(2)$ \\
For other anxiety problem & $15(3)$ & $20(4)$ \\
For another non-anxiety problem & $20(4)$ & $15(3)$ \\
Treated with medication & $0(0)$ & $5(1)$ \\
\hline
\end{tabular}

There were no significant between-group differences for any of the above characteristics at baseline

$C B T$ cognitive behavior therapy, $G A D$ generalized anxiety disorder severe learning difficulties were excluded from the study. Two participants had a statement of special educational need, one tied to a comorbid diagnosis of ADHD (randomized to CBT) and one tied to a motor disorder (randomized to WL). No other participants had a diagnosis of neurodevelopmental disorder or statement of educational need. No standardized measure of socioeconomic status was used in the study. Reflecting the socioeconomic diversity of the catchment area from which all participants were recruited, 10 of the 40 participants $(25 \%)$ were living in a home where the parents were receiving either income or housing benefits (6 in CBT, 4 in WL).

\section{Primary and Secondary Outcomes (Assessor/ Clinician-Rated)}

Table 2 present the proportion of participants in the CBT and WL groups meeting criteria for GAD at pre- and posttreatment (primary outcome), and for CBT participants (only) at the 3-month follow-up, and secondary assessor/ clinician-rated secondary outcomes. At post-treatment/WL, CBT participants were more likely than WL participants to have remitted from their GAD diagnosis, as well from the comorbid disorders that were preset at pre-treatment/WL. Significant between group differences at post-treatment/ $\mathrm{WL}$, in the large range $\left(\eta^{2} \geq .26\right)$, were found for assessor's blinded ratings of GAD severity (ADIS-C/P) and clinicianrated global functioning (CGAS). Age and gender of the participant at pre-treatment were unrelated to likelihood of remission GAD (or comorbid diagnoses) at post-treatment or 3-month follow-up for treated participants.

Within-subject analyses were carried out for the CBT participants (pre-, post-treatment, and follow-up). As noted above, significant pre-to-post treatment reductions were observed in the proportion of CBT participants with a GAD diagnosis. No further changes occurred in this variable between post-treatment and month follow-up. Significant time effects, in the large range, were observed for GAD severity (ADIS-C/P; F $(2,18)=44.8, \mathrm{p}=.000, \eta^{2}=.78$ ) and Global functioning (CGAS: $\mathrm{F}(2,18)=34.6, \mathrm{p}=.000$, $\eta^{2}=.77$ ). Post-hoc comparisons (Bonferroni) revealed significant differences between pre- and post-treatment, between pre-treatment and follow-up only (all p's $=.000$ ).

\section{Secondary Outcomes (Child/Parent-Report)}

Table 3 presents results for the secondary outcomes (childand parent-reports). Significant differences, in the large range of effect sizes, were observed between the CBT and WL groups at post-treatment/WL for all secondary outcomes except conduct problems (parent-report). The results of the within subject analyses for CBT participants on the child/ parent-report secondary outcomes and cognitive measures 
Table 2 Intention-to-treat, primary and secondary, assessor- and clinician-rated outcomes for treated and wait-listed participants

\begin{tabular}{|c|c|c|c|c|c|c|c|}
\hline \multirow[t]{2}{*}{ Assessor/clinician-rated outcomes } & \multicolumn{3}{|l|}{$\begin{array}{l}\text { CBT } \\
(n=20)\end{array}$} & \multicolumn{2}{|l|}{$\begin{array}{l}\text { Wait-list } \\
(\mathrm{n}=20)\end{array}$} & \multicolumn{2}{|c|}{$\begin{array}{l}\text { CBT vs wait-list } \\
\text { at post-treatment }\end{array}$} \\
\hline & Pre & Post & 3 Mos. FUP & Pre & Post & $X^{2}, F$ & $\eta^{2}$ \\
\hline \multicolumn{8}{|l|}{ Primary outcome } \\
\hline GAD diagnosis present, \% (n) & $100(20)$ & $20(4)$ & $15(3)$ & $100(20)$ & $100(20)$ & $26.7 * * *$ & \\
\hline \multicolumn{8}{|l|}{ Secondary outcomes } \\
\hline GAD severity rating, $M(S D)$ & $6.8(1.1)$ & $1.9(2.3)$ & $1.9(2.6)$ & $6.9(1.1)$ & $5.7(1.1)$ & $42.5 * * *$ & .54 \\
\hline Comorbid disorder present, $\%$ (n) & $60(12)$ & $5(1)$ & $5(1)$ & $55(11)$ & $55(11)$ & $11.9 * * *$ & \\
\hline Recovered from all disorders, $\%$ (n) & $0(20)$ & $80(16)$ & $90(18)$ & $0(20)$ & $0(20)$ & $26.7 * * *$ & \\
\hline Global functioning, $M(S D)$ & $56.6(7.9)$ & $82.1(8.9)$ & $82.7(9.9)$ & $59.7(6.8)$ & $59.4(6.7)$ & $87.2 * * *$ & .70 \\
\hline
\end{tabular}

Diagnostic status (GAD and comorbid disorders) and GAD severity were assessed by independent assessors (blinded to allocation) using the anxiety disorders interview schedule for DSM-IV-child and parent versions; Global functioning was assessed by the clinician using the child global assessment of functioning scale (CGAS); There were significant pre-to-post-treatment and pre-to-follow-up time effects in the CBT group for GAD severity and global functioning

CBT cognitive behavior therapy, FUP Follow-up

$* * * \mathrm{p}<.000$

Table 3 Intention to treat, child- and parent-reported secondary outcomes, for treated and wait-listed participants

\begin{tabular}{|c|c|c|c|c|c|c|c|}
\hline \multirow[t]{2}{*}{ Measure } & \multicolumn{3}{|l|}{$\begin{array}{l}\text { CBT } \\
(n=20)\end{array}$} & \multicolumn{2}{|l|}{$\begin{array}{l}\text { Wait-list } \\
(\mathrm{n}=20)\end{array}$} & \multicolumn{2}{|c|}{$\begin{array}{l}\text { CBT versus } \\
\text { wait-list at } \\
\text { post-treatment }\end{array}$} \\
\hline & Pre M (SD) & Post M (SD) & 3 Mos. FUP M (SD) & Pre M (SD) & Post M (SD) & $X^{2}, F$ & $\eta^{2}$ \\
\hline \multicolumn{8}{|l|}{ Secondary outcomes (child-report) } \\
\hline Worry $(P S W Q-C)$ & $33.6(5.4)$ & $10.6(12.2)$ & $10.8(13.2)$ & $34.4(5.3)$ & $31.1(7.2)$ & $43.6^{* * *}$ & .54 \\
\hline Anxiety $(S C A R E D-R-C)$ & $41.2(13.3)$ & $15.2(12.5)$ & $16.8(14.9)$ & $48.9(11.6)$ & $46.3(15.9)$ & $40.9 * * *$ & .53 \\
\hline GAD (SCARED-R-C-GAD) & $11.9(3.0)$ & $4.6(5.2)$ & $4.8(5.2)$ & $13.5(3.5)$ & $12.9(4.2)$ & $32.5^{* * *}$ & .47 \\
\hline Depression $(M F Q-C)$ & $26.5(13.9)$ & $6.9(9.8)$ & $8.6(10.8)$ & $27.0(11.2)$ & $25.4(14.4)$ & $24.9 * * *$ & .40 \\
\hline Quality of life $(P Q-L E S-Q)$ & $48.9(7.1)$ & $60.8(10.7)$ & $59.1(12.5)$ & $45.6(6.0)$ & $48.7(9.4)$ & $11.3 * *$ & .23 \\
\hline Intolerance of uncertainty (Brief-IUS) & $18(5.9)$ & $9.7(5.5)$ & $9.1(5.3)$ & $17.1(4.1)$ & $17.7(5.0)$ & $34.7 * * *$ & .48 \\
\hline Cognitive avoidance (Brief-CAQ) & $15.8(4.5)$ & $9.9(4.6)$ & $11.2(4.5)$ & $18.7(3.2)$ & $18.1(4.7)$ & $22.6 * * *$ & .38 \\
\hline Negative problem orientation (Brief-NPO) & $17.2(5.7)$ & $8.1(4.1)$ & $8.5(4.6)$ & $16.4(4.8)$ & $15.3(6.3)$ & $20.8 * * *$ & .36 \\
\hline Positive beliefs about worry (Brief- $W W$-II) & $10.3(3.7)$ & $8.3(4.2)$ & $7.7(4.2)$ & $10.2(4.1)$ & $9.5(4.9)$ & 1.0 & .03 \\
\hline \multicolumn{8}{|l|}{ Parent-report } \\
\hline Anxiety $(S C A R E D-R-P)$ & $41.5(15.1)$ & $18.9(12.4)$ & $19.7(4.8)$ & $42.6(13.7)$ & $38.2(14.9)$ & $21.5^{* * *} *$ & .37 \\
\hline GAD (SCARED-R-P-GAD) & $11.9(3.8)$ & $6.5(4.3)$ & $6.4(4.9)$ & $12.6(3.1)$ & $11.2(4.7)$ & $11.7 * *$ & .24 \\
\hline Depression $(M F Q-P)$ & $24.1(12.0)$ & $10.1(9.7)$ & $11.8(9.8)$ & $26.8(15.0)$ & $20.9(14.9)$ & $8.4 * *$ & .19 \\
\hline Conduct problems (SDQ-P-conduct) & $2.4(1.5)$ & $1.9(1.6)$ & $1.8(1.3)$ & $1.9(1.5)$ & $1.7(1.5)$ & .04 & .00 \\
\hline Peer difficulties (SDQ-P-peer) & $3.9(2.2)$ & $1.7(1.6)$ & $2.0(2.2)$ & $3.2(1.9)$ & $2.6(1.9)$ & $8.6^{* *}$ & .19 \\
\hline Overall impairment (SDQ-P-impairment) & $6.2(3.1)$ & $1.7(2.2)$ & $2.0(2.6)$ & $5.5(3.4)$ & $4.4(3.4)$ & $11.5^{* *}$ & .23 \\
\hline
\end{tabular}

All means are for total scores on the scale/subscale

A significant main effect for time was observed for the CBT group for all outcomes except conduct problems, with significant differences for pre-treatment versus post-treatment and 3-month follow-up, but no differences between post-treatment and follow-up in the CBT group

$C B T$ cognitive behavior therapy, $W L$ wait-list, $F U P$ follow-up, $P S W Q-C$ penn state worry questionnaire for children, $S C A R E D-R-C / P$ child anxiety related emotional disorders-child/parent version, $M F Q-C$ mood and feelings questionnaire-child/parent version, $P Q-L E S-Q$ pediatric quality of life enjoyment and satisfaction questionnaire, Brief-IUS brief intolerance of uncertainty scale, Brief-CAQ brief cognitive avoidance scale, Brief-NPO brief negative problem orientation scale, Brief-WW-II brief why worry scale II, SDQ-P strengths and difficulties questionnaire-parent version

$* * \mathrm{p}<.01, * * * \mathrm{p}<.001$ 
are not reported in Table 3. A significant effect for time was observed for all child-reported outcomes, including: worry (PSWQ-C: $\mathrm{F}(2,18)=61.1, \mathrm{p}=.000, \eta^{2}=.76$ ); total anxiety (SCARED-C: $\left.\mathrm{F}(2,38)=39.8, \mathrm{p}=.000, \eta^{2}=.67\right)$; GAD symptoms (SCARED-C-GAD: $\mathrm{F}(2,38)=37.0, \mathrm{p}=.000$, $\eta^{2}=.61$ ); depression (MFQ-C: $\mathrm{F}(2,38)=24.4, \mathrm{p}=.000$, $\eta^{2}=.60$ ); and quality of life (PQ-LES-Q: $\mathrm{F}(2,38)=26.9$, $\mathrm{p}=.000, \eta^{2}=.59$ ). Post-hoc comparisons (Bonferroni) revealed significant differences between pre- and posttreatment, and between pre-treatment and follow-up only (all p's $=.000)$.

Significant time effects were also observed for parentreported, secondary outcomes including: total anxiety (SCARED-P Total: $\left.\mathrm{F}(2,38)=29.1, \mathrm{p}=.000, \mathrm{n}^{2}=.61\right)$; GAD symptoms (SCARED-P-GAD: $F(2,38)=20.5$, $\mathrm{p}=.000, \mathrm{\eta}^{2}=.52$ ); depression (MFQ-P: $\mathrm{F}(2,38)=20.5$, $\left.\mathrm{p}=.000, \mathrm{n}^{2}=.52\right)$; difficulties with peers (SDQ-P-Peer: $\mathrm{F}(2$, $\left.38)=31.1, \mathrm{p}=.000, \mathrm{\eta}^{2}=.62\right)$; overall impairment (SDQ-PImpairment: $\left.F(2,38)=30.9, p=.000, \eta^{2}=.62\right)$. No time effect was found for parent-reported conduct problems (SDQ-P-Behavioral: $\mathrm{F}(2,38)=2.5, \mathrm{p}=.09, \mathrm{\eta}^{2}=.12$ ). Again, post hoc comparisons (Bonferroni) revealed significant differences between pre- and post-treatment, and between pretreatment and follow-up only (all p's $=.000$ ).

\section{Cognitive Process Measures}

Table 4 presents results for the cognitive process measures (child-report). Significant differences, in the large range of effect sizes, were observed between the CBT and WL groups at post-treatment/WL for intolerance of uncertainty, cognitive avoidance, and negative problem orientation, but not positive beliefs about worry. For the within-subject analyses (not reported in Table 4) for the CBT participants, significant effects for time was observed for intolerance of uncertainty (Brief-IUS: $\mathrm{F}(2,18)=19.9, \mathrm{p}=.000, \mathrm{\eta}^{2}=.62$ ), cognitive avoidance (Brief-CAQ: $\mathrm{F}(2,18)=10.9, \mathrm{p}=.001$, $\eta^{2}=.41$ ), and negative problem orientation (Brief-NPO: $\left.\mathrm{F}(2,18)=19.4, \mathrm{p}=.000, \mathrm{\eta}^{2}=.61\right)$, but not positive beliefs about worry (Brief-WW-II: $\mathrm{F}(2,18)=7.1, \mathrm{p}=.06, \mathrm{\eta}^{2}=.27$ ). For intolerance of uncertainty, cognitive avoidance, and negative problem orientation, post hoc comparisons (Bonferroni) revealed significant differences between pre- and post-treatment, and between pre-treatment and follow-up only (all p's $=.000$ ).

\section{Discussion}

This study was designed as a randomized controlled, pilot feasibility trial with the aim of assessing the tolerability and efficacy of an individual, child-focused, GAD-specific CBT under randomized controlled conditions. The treatment is based on (and targets) four cognitive processes from the Laval model of worry: intolerance of uncertainty, cognitive avoidance, negative problem orientation, and positive beliefs about worry. The secondary aim was to assess whether changes in these four processed occurred for the treated versus the wait-listed participants. This is only the second controlled trial of GAD-specific CBT with children and the first with adolescents. It is the third trial to report change scores on measures of the four cognitive processes from the Laval model.

The treatment was well tolerated with only two noncompleters, both adolescents. One of whom removed themselves from the trial because they wanted to focus more on their symptoms of social anxiety disorder. The other was removed from the trial due to an acute onset suicidal depression relating to a family crisis, not their GAD diagnosis or treatment. Using a measure developed

Table 4 Intention to treat, child-reported cognitive process measures, for treated and wait-listed participants

\begin{tabular}{|c|c|c|c|c|c|c|c|}
\hline \multirow[t]{2}{*}{ Cognitive process measure } & \multicolumn{3}{|c|}{ CBT $(n=20)$} & \multicolumn{2}{|c|}{ Wait-list $(\mathrm{n}=20)$} & \multicolumn{2}{|c|}{$\begin{array}{l}\text { CBT versus } \\
\text { wait-list at } \\
\text { post-treatment }\end{array}$} \\
\hline & Pre M (SD) & Post M (SD) & 3 Mos. FUP M (SD) & Pre M (SD) & Post M (SD) & $X^{2}, F$ & $\eta^{2}$ \\
\hline Intolerance of uncertainty (Brief-IUS) & $18(5.9)$ & $9.7(5.5)$ & $9.1(5.3)$ & $17.1(4.1)$ & $17.7(5.0)$ & $34.7 * * *$ & .48 \\
\hline Cognitive avoidance (Brief-CAQ) & $15.8(4.5)$ & $9.9(4.6)$ & $11.2(4.5)$ & $18.7(3.2)$ & $18.1(4.7)$ & $22.6 * * *$ & .38 \\
\hline Negative problem orientation (Brief-NPO) & $17.2(5.7)$ & $8.1(4.1)$ & $8.5(4.6)$ & $16.4(4.8)$ & $15.3(6.3)$ & $20.8 * * *$ & .36 \\
\hline Positive beliefs about worry (Brief- $W W-I I$ ) & $10.3(3.7)$ & $8.3(4.2)$ & $7.7(4.2)$ & $10.2(4.1)$ & $9.5(4.9)$ & 1.0 & .03 \\
\hline
\end{tabular}

All means are for total scores on the scales

For treated participants, significant pre-to-post-treatment and pre-treatment-to-follow-up effects for time were observed for intolerance of uncertainty, cognitive avoidance, and negative problem orientation

$C B T$ cognitive behavior therapy, FUP follow-up, Brief-IUS brief intolerance of uncertainty scale, Brief-CAQ brief cognitive avoidance scale, Brief-NPO brief negative problem orientation scale, Brief-WW-II brief why worry scale II

$* * * \mathrm{p}<.001$ 
for this trial, the participants reported that the treatment was highly credible, and they felt able to engage well with their therapist. Including the present study, three trials have been carried out with children between seven and 12 years of age using GAD-specific CBT targeting the four cognitive processes from the Laval model, with less than $15 \%$ drop-outs, and high rates of recovery (Holmes et al. 2014; Payne et al. 2011). While further research is needed, the findings from the three trials suggest that children and adolescents are able to engage with a treatment focused largely on cognitive processes associated with worry and GAD severity.

For the primary outcome variable, measured according to intention-to-treat, a large and significant difference was observed between the CBT and wait-listed participants for GAD remission ( $80 \%$ vs $0 \%$, respectively) There was a slight but non-significant increase in the proportion of CBT participants with GAD at follow-up (85\%), suggesting that improvements at the diagnostic threshold level were largely maintained. These findings are similar to the intention-totreat outcomes for GAD remission reported for post-treatment in our uncontrolled pilot of the same treatment $(81 \%$; Payne et al. 2011), and at the 3 month-follow up (80\%; intention-to-treat) in the randomized controlled trial of a group-based treatment (Holmes et al. (2014). The present findings for GAD remission rates are also comparable to those reported in trials of adults with GAD treated with the CBT approach (Dugas and Robichaud 2007) on which the current treatment is based (70-88\%; Ladouceur et al. 2000, 2004; Dugas et al. 2003, 2010).

Consistent with Payne et al. (2011) and Holmes et al. (2014), the effects of the present treatment were not limited to remission from GAD. Significant reductions were observed in the rate of comorbid disorders, as well as moderate to large effects for child- and parent-reported worry, anxiety, depression, peer problems, overall impairment, and quality of life. These gains were maintained at the 3-month follow-up for treated participants.

The present study was also designed to assess whether changes in GAD and worry would be accompanied by changes in the four cognitive processes that are targeted in treatment. Consistent with the Laval model, and expectation, treated participants experienced large reductions in IU, cognitive avoidance, and negative problem orientation. However, no significant changes were found for positive beliefs about worry. The same pattern of findings for the four cognitive processes were reported by both Holmes et al. (2014) and Wahlund et al. (2019). This trial was not designed to test for mediation and so it not possible to conclude that changes in the primary or secondary outcomes occurred because of changes in IU, cognitive avoidance, or negative problem orientation, or that the interventions themselves were responsible for any observed changes in these processes. What the present findings, and those of previous GAD/IU-focused treatments with youth, do suggest is that trials capable of assessing whether changes in the four cognitive processes mediate outcomes are warranted.

The absence of findings for positive beliefs about worry in the present trial requires further comment. It is possible that the lack of change in this process during treatment, not just in this trial but also in Holmes et al. (2014) and Wahlund et al. (2019), reflects a problem with the way that positive beliefs about worry were measured. Both this trial and Wahlund et al. (2019), used a 5-item version of the 25-item, Why Worry Scale-II scale for adults (Holowka et al. 2000). Holmes et al. (2014) used a 6-item subscale from the Metacognitions Questionnaire for Children (Bacow et al. 2009). While scores on these measures of positive beliefs about worry correlate in the moderate to large range with childand adolescent-reported worries and anxiety (Bacow et al. 2009; Fialko et al. 2012), it is possible that neither scale is sufficiently sensitive to positive beliefs about worry for treatment purposes. At present, there is no consensus in the literature on the measurement of IU, cognitive avoidance, negative problem orientation, or positive beliefs about worry (in adults or children). Further, scale development, and validation with children and adolescents, is necessary before any conclusions can be drawn about the importance of targeting positive beliefs about worry in young people with GAD.

As a pilot examination of the tolerability and efficacy of a GAD-specific treatment for youth, the study benefitted from several strengths, including: a randomized controlled design; a manualized treatment; primary outcomes based on blinded, structured diagnostic interviews; a broad range of standardized, secondary outcome measures obtained from multiple informants; and the use of measures designed to assess processes assumed to underlie change in treatment. This is only the second randomized controlled trial of a GAD-specific treatment carried out with children and the first with adolescents. Nevertheless, the findings must be viewed within the context of certain limitations. While a comparison with wait-list controls, and a brief follow-up for treated participants, are appropriate design elements for a pilot feasibility trial, studies involving active treatment and placebo controls, and longer follow-ups are are needed to properly estimate the efficacy of the present treatment. Second, while the treatment was manualized, and the therapists trained and supervised in its use, no formal treatment adherence protocol was followed. Third, interrater reliability ratings were not obtained for the diagnostic assessments. Fourth, the treatment credibility and cognitive process measures were developed specifically for this trial and required further examination of their validity in clinical samples. Finally, treatment was delivered in a specialist anxiety disorders clinic by experienced therapists receiving weekly supervision. The treatment should also be evaluated 
in routine care settings where most anxiety disordered children are seen.

In conclusion, the present findings add to a small body of literature suggesting that GAD-specific CBT treatments are acceptable to children as young as 7 years and adolescents up to 19 years. In uncontrolled and controlled trials involving wait-list controls, these treatments appear to yield high rates of remission from GAD and comorbid disorders, as well as moderate to large reductions in worry, anxiety, depressive symptoms, and overall functioning. The findings also add to a small body of evidence suggesting that IU, cognitive avoidance, negative problem orientation, and positive beliefs are associated with the severity of worry, anxiety and GAD in youth, and important targets for further investigation in a clinical context.

Acknowledgements The authors express their sincere gratitude to the participating families. We would like to thank the reviewers for their comments on an earlier version of the manuscript.

Funding This work was supported by a grant from the National Institute of Health Research to S. Perrin and D. Bolton (NIHR; 080817094), and with additional supports from the Guy's \& St Thomas' Charity (EFT 120604), and the NIHR Biomedical Research Centre at the South London and Maudsley NHS Foundation Trust and King's College London. The views expressed are those of the authors and not necessarily those of the NHS, the NIHR, or the Department of Health.

\section{Compliance with Ethical Standards}

Conflict of Interest Sean Perrin, Denise Bevan, Susanna Payne, and Derek Bolton declare that they have no conflicts of interest.

Ethical Approval This study was approved by the National Health Service Research Ethics Committee (ref: 09/H0720/127) and Institutional Review Board of the South London and Maudsley NHS Foundation Trust. All procedures were performed in accordance with the ethical standards of the institution, the research ethics committee, and the 1964 Helsinki declaration, and its later amendments, on research with human subjects.

Informed Consent Written informed consent was obtained from the participants and at least one parent or legal guardian. No financial incentive was offered for participation.

Animal Rights No animals studies were carried out by the authors for this article.

Open Access This article is distributed under the terms of the Creative Commons Attribution 4.0 International License (http://creativeco mmons.org/licenses/by/4.0/), which permits unrestricted use, distribution, and reproduction in any medium, provided you give appropriate credit to the original author(s) and the source, provide a link to the Creative Commons license, and indicate if changes were made.

\section{References}

American Psychiatric Association. (1994). Diagnostic and statistical manual of mental disorders (4th ed.). Washington, DC: APA.

American Psychiatric Association. (2013). Diagnostic and statistical manual of mental disorders (5th ed.). Washington, DC: APA.

Angold, A., Costello, E. J., Messer, S. C., Pickles, A., Winder, F., \& Silver, D. (1995). The development of a short questionnaire for use in epidemiological studies of depression in children and adolescents. International Journal of Methods in Psychiatric Research, 5, 237-249.

Bacow, T. L., Pincus, D. B., Ehrenreich, J. T., \& Brody, L. R. (2009). The metacognitions questionnaire for children: Development and validation in a clinical sample of children and adolescents with anxiety disorders. Journal of Anxiety Disorders, 23(6), 727-736.

Birmaher, B., Khetarpal, S., Brent, D., Cully, M., Balach, L., Kaufman, J., et al. (1997). The screen for child anxiety related emotional disorders (SCARED): Scale construction and psychometric characteristics. Journal of the American Academy of Child and Adolescent Psychiatry, 4, 545-553.

Bomyea, J., Ramsawh, H., Ball, T. M., Taylor, C. T., Paulus, M. P., Lang, A. J., et al. (2015). Intolerance of uncertainty as a mediator of reductions in worry in a cognitive behavioral treatment program for generalized anxiety disorder. Journal of Anxiety Disorders, $33,90-94$

Borkovec, T. D., Newman, M. G., Pincus, A. L., \& Lytle, R. (2002). A component analysis of cognitive-behavioral therapy for generalised anxiety disorder and the role of interpersonal problems. Journal of Consulting and Clinical Psychology, 70, 288-298.

Borkovec, T. D., Newman, M. G., \& Castonguay, L. G. (2003). Cognitive behavioural therapy for generalized anxiety disorder with integrations from interpersonal and experiential therapies. CNS Spectrums, 8, 382-389.

Carleton, R. N. (2016). Into the unknown: A review and synthesis of contemporary models involving uncertainty. Journal of Anxiety Disorders, 39, 30-43.

Chorpita, B. F., Tracey, S. A., Brown, T. A., Colica, T. J., \& Barlow, D. H. (1997). Assessment of worry in children and adolescents: An adaptation of the Penn State Worry Questionnaire. Behaviour Research and Therapy, 35, 569-581.

Cohen, J. (1988). Statistical power analysis for the behavioral sciences. New Jersey: Routledge.

Copeland, W. E., Angold, A., Shanahan, L., \& Costello, E. J. (2014). Longitudinal patterns of anxiety from childhood to adulthood: The great smoky mountains study. Journal of the American Academy of Child and Adolescent Psychiatry, 53(1), 21-33.

Devilly, G. J., \& Borkovec, T. D. (2000). Psychometric properties of the credibility/expectancy questionnaire. Journal of Behavior Therapy and Experimental Psychiatry, 31, 71-86.

Donovan, C. L., Holmes, M. C., \& Farrell, L. J. (2016). Investigation of the cognitive variables associated with worry in children with generalised anxiety disorder and their parents. Journal of Affective Disorders, 192, 1-7.

Dugas, M., \& Robichaud, M. (2007). Cognitive-behavioural therapy for generalized anxiety disorder. New York: Routledge.

Dugas, M. J., Gagnon, F., Ladouceur, R., \& Freeston, M. H. (1998) Generalized anxiety disorder: A preliminary test of a conceptual model. Behavior Research and Therapy, 36, 215-226.

Dugas, M. J., Ladouceur, R., Leger, E., Freeston, M. H., Langlois, F., Provencher, M. D., et al. (2003). Group cognitive-behavioral therapy for generalized anxiety disorder: Treatment outcome and long-term follow-up. Journal of Consulting and Clinical Psychology, 4, 821-825.

Dugas, M. J., Brillon, P., Savard, P., Turcotte, J., Gaudet, A., Ladouceur, R., et al. (2010). A randomized clinical trial of 
cognitive-behavioural therapy and applied relaxation for adults with generalized anxiety disorder. Behavior Therapy, 41(1), $46-58$.

Dugas, M. J., Laugesen, N., \& Bukowski, W. M. (2012). Intolerance of uncertainty, fear of anxiety, and adolescent worry. Journal of Abnormal Child Psychology, 40(6), 863-870.

Eisen, A., \& Silverman, W. K. (1993). Should I relax or change my thoughts? A preliminary examination of cognitive therapy, relaxation training, and their combination with overanxious children. Journal of Cognitive Psychotherapy, 7, 265-279.

Eisen, A. R., \& Silverman, W. K. (1999). Prescriptive treatment for generalized anxiety disorder in children. Behavior Therapy, 29(1), $105-121$.

Endicott, J., Nee, J., Yang, R., \& Wohlberg, C. (2006). Pediatric quality of life enjoyment and satisfaction questionnaire (PQ-LES-Q): Reliability and validity. Journal of the American Academy of Child and Adolescent Psychiatry, 45(4), 401-407.

Ewing, D. L., Monsen, J. J., Thompson, E. J., Cartwright-Hatton, S., \& Field, A. (2015). A meta-analysis of transdiagnostic cognitive behavioural therapy in the treatment of child and young person anxiety disorders. Behavioural \& Cognitive Psychotherapy, 43(5), $562-577$.

Fialko, L. (2009). Cognitive variables associated with worry in young people aged 7-18 (Doctoral dissertation). Institute of Psychiatry, Psychology \& Neuroscience, King's College London.

Fialko, L., Bolton, D., \& Perrin, S. (2012). Applicability of a cognitive model of worry to children and adolescents. Behaviour Research and Therapy, 50(5), 341-349.

Freeston, M. H., Rhéaume, J., Letarte, H., Dugas, M. J., \& Ladouceur, R. (1994). Why do people worry? Personality and Individual Differences, 17, 791-802.

Goodman, R. (1997). The strengths and difficulties questionnaire: A research note. Journal of Child Psychology and Psychiatry, 38, $581-586$.

Holmes, M. C., Donovan, C. L., Farrell, L. J., \& March, S. (2014). The efficacy of a group- based, disorder-specific treatment program for childhood GAD: A randomized controlled trial. Behavior Research and Therapy, 61, 122-135.

Holowka, D. W., Dugas, M. J., Francis, K., \& Laugesen, N. (2000). Measuring beliefs about worry: A psychometric evaluation of the Why Worry-II Questionnaire. Poster presented at the Association for Advancement of Behavior Therapy's 34th Annual Convention, New Orleans, LA.

Hudson, J. L., Rapee, R. M., Lyneham, H. J., McLellan, L. F., Wuthrich, V. M., \& Schniering, C. A. (2015). Comparing outcomes for children with different anxiety disorders following cognitive behavioural therapy. Behavior Research and Therapy, 72(9), 30-37.

James, A. C., James, G., Cowdrey, F. A., Soler, A., \& Choke, A. (2013). Cognitive behavioural therapy for anxiety disorders in children and adolescents. Cochrane Database Systematic Review. https:// doi.org/10.1002/14651858.CD004690.pub3.

Kane, M. T., \& Kendall, P. C. (1989). Anxiety disorders in children: A multiple-baseline evaluation of a cognitive-behavioral treatment. Behavior Therapy, 20, 499-508.

Kendall, P. C. (1990). The coping cat manual. Ardmore PA: Workbook Publishing.

Kendall, P. C., Compton, S. N., Walkup, J. T., Birmaher, B., Albano, A. A., Sherrill, J., et al. (2010). Clinical characteristics of anxiety disordered youth. Journal of Anxiety Disorders, 24(3), 360-365.

Ladouceur, R., Dugas, M., Freeston, M. H., Leger, E., Gagnon, F., \& Thibodeau, N. (2000). Effectiveness of a new cognitive-behavioral treatment for generalized anxiety disorder: Evaluation in a controlled clinical trial. Journal of Consulting and Clinical Psychology, 68, 957-964.
Ladouceur, R., Léger, É., Dugas, M., \& Freeston, M. (2004). Cognitive-behavioral treatment of generalized anxiety disorder (GAD) for older adults. International Psychogeriatrics, 16(2), 195-207.

Laugesen, N., Dugas, M. J., \& Bukowski, W. M. (2003). Understanding adolescent worry: The application of a cognitive model. Journal of Abnormal Child Psychology, 31, 55-64.

Leger, E., Ladouceur, R., Dugas, M. J., \& Freeston, M. H. (2003). Cognitive-behavioural treatment of generalized anxiety disorder among adolescents: A case series. Journal of the American Academy of Child and Adolescent Psychiatry, 42, 327-330.

Lyneham, H. J., Abbott, M. J., Wignall, A., \& Rapee, R. M. (2003). The cool kids anxiety treatment program. Sydney, Australia: MUARU, Macquarie University.

Mufson, L., Moreau, D., \& Weissman, M. M. (1994). Modification of interpersonal psychotherapy with depressed adolescents (IPT-A): Phase I and phase II studies. Journal of the American Academy of Child and Adolescent Psychiatry, 33(5), 695-705.

Osmanağaoğlu, N., Creswell, C., \& Dodd, H. F. (2018). Intolerance of uncertainty, anxiety, and worry in children and adolescents: A meta-analysis. Journal of Affective Disorders, 225, 80-90.

Payne, S., Bolton, D., \& Perrin, S. (2011). A pilot investigation of cognitive therapy for generalized anxiety disorder in children aged 7-17 years. Cognitive Therapy \& Research, 25(2), 171-178.

Robichaud, M., \& Dugas, M. J. (2005). Negative problem orientation (Part I): Psychometric properties of a new measure. Behaviour Research and Therapy, 43(3), 391-401.

Sexton, K. A., \& Dugas, M. J. (2008). The cognitive avoidance questionnaire: Validation of the english translation. Journal of Anxiety Disorders, 22(3), 355-370.

Shaffer, D., Gould, M. S., Brasic, J., Ambrosini, P., Fisher, P., Bird, H., et al. (1983). A children's global assessment scale (CGAS). Archives of General Psychiatry, 40(11), 1228-1231.

Silverman, W., \& Nelles, W. (1998). The anxiety disorders interview schedule for children. Journal of the American Academy of Child and Adolescent Psychiatry, 27, 772-778.

Torbit, L., \& Laposa, J. M. (2016). Group CBT for GAD: The role of change in intolerance of uncertainty in treatment outcomes. International Journal of Cognitive Therapy, 9(4), 356-368.

Uhmann, S., Beesdo-Baum, K., Becker, E. S., \& Hoyer, J. (2010). Specificity of interpersonal problems in generalized anxiety disorder versus other anxiety disorders and depression. Journal of Nervous \& Mental Disorders, 198(11), 846-851.

Wahlund, T., Andersson, E., Jolstedt, M., Perrin, S., Vigerland, S., \& Serlachius, E. (2019). Intolerance of uncertainty-focused treatment for adolescents with excessive worry-a pilot feasibility study. Cognitive \& Behavioral Practice.

Waite, P., \& Creswell, C. (2014). Children and adolescents referred for treatment of anxiety disorders: Differences in clinical characteristics. Journal of Affective Disorders, 167(100), 326-332.

Wang, Z., Whiteside, S. P., Sim, L., Farah, W., Morrow, A. S., Alsawas, M., et al. (2017). Comparative effectiveness and safety of cognitive behavioral therapy and pharmacotherapy for childhood anxiety disorders: A systematic review and meta-analysis. JAMA Pediatrics, 171(11), 1049-1056.

Waters, A. M., Donaldson, J., \& Zimmer-Gembeck, M. J. (2008). Cognitive-behavioural therapy combined with an interpersonal-skills component in the treatment of generalised anxiety disorder in adolescent females: A case series. Behaviour Change, 25(1), 35-43.

Publisher's Note Springer Nature remains neutral with regard to jurisdictional claims in published maps and institutional affiliations. 